\title{
GCU
}

Glasgow Caledonian

University

University for the Common Good

\section{Development of an Alcohol Withdrawal risk stratification tool based on patients referred to an addiction liaison nursing service in Glasgow}

Benson, George; McPherson, Andrew; McCallum, Jacqueline; Roberts, Nicola

Published in:

Drugs and Alcohol today

DOI:

10.1108/DAT-02-2019-0009

Publication date:

2019

Document Version

Author accepted manuscript

Link to publication in ResearchOnline

Citation for published version (Harvard):

Benson, G, McPherson, A, McCallum, J \& Roberts, N 2019, 'Development of an Alcohol Withdrawal risk stratification tool based on patients referred to an addiction liaison nursing service in Glasgow', Drugs and Alcohol today, vol. 19, no. 4, pp. 241-250. https://doi.org/10.1108/DAT-02-2019-0009

\section{General rights}

Copyright and moral rights for the publications made accessible in the public portal are retained by the authors and/or other copyright owners and it is a condition of accessing publications that users recognise and abide by the legal requirements associated with these rights.

Take down policy

If you believe that this document breaches copyright please view our takedown policy at https://edshare.gcu.ac.uk/id/eprint/5179 for details of how to contact us. 


\title{
DEVELOPMENT OF AN ALCOHOL WITHDRAWAL RISK STRATIFICATION TOOL BASED ON PATIENTS REFERRED TO AN ADDICTION LIAISON NURSING SERVICE IN GLASGOW
}

\begin{abstract}
Purpose: To develop an Alcohol Withdrawal Syndrome (AWS) risk stratification tool that could support the safe discharge of low risk patients from the emergency department.

Design/Methods: A retrospective cohort study that included all patients referred to the acute addiction liaison nursing service (AALNS) over one calendar month ( $\mathrm{n}=400,1^{\text {st }}$ April 2016$30^{\text {th }}$ April 2016) was undertaken. Bivariate and multivariate modelling identified the significant variables that supported the prediction of severe alcohol withdrawal syndrome (SAWS) in the cohort population.

Findings: The Glasgow Modified Alcohol Withdrawal Scale (GMAWS), hours since last drink, Fast Alcohol Screening Test (FAST), and systolic blood pressure (SBP) correctly identified $89 \%$ of patients who developed SAWS and $84 \%$ of patients that did not. Increasing each component by a score of one is associated with an increase in the odds of SAWS by a factor of 2.76 (95\% CI 2.21, 3.45), 1.31 (95\% CI 1.24, 1.37), 1.30 (CI 1.08, 1.57) and 1.22 $(95 \%$ CI $1.10,1.34)$ respectively.
\end{abstract}

Research Limitations: The research was conducted in a single healthcare system that had a high prevalence of alcohol dependence syndrome (ADS). Second, the developed risk stratification tool was unable to guarantee no risk and lastly, the FAST score previously aligned to severe ADS may have influenced the patients highest GMAWS score.

Originality/ Value: The tool could help redesign the care pathway for emergency department patients at low risk of SAWS and link them with community alcohol services better equipped to deal with their physical and psychological needs, short and long term, supporting engagement, abstinence and prolongation of life.

KEYWORDS: alcohol, general hospital, delirium tremens, alcohol-related seizures, alcohol withdrawal syndrome, risk prediction, hospital admission, risk stratification 


\section{INTRODUCTION}

Alcohol use is one of the major causes of disease, death and injury globally (World Health Organisation, 2014). Excessive alcohol consumption is linked to over 200 physical and psychological diseases and has a longstanding association with cardiovascular disease, liver cirrhosis and various cancers (World Health Organisation, 2014; Holman et al., 1996). From an acute health perspective the greatest impact of problematic alcohol use is witnessed by emergency departments, where at peak times $70 \%$ of attendances are alcohol related (Vardy et al., 2017; Waye et al., 2015; Stehman \& Mycyk, 2013; Manasco. et al., 2012; Forsythe \& Lee, 2012; The Department of Health, 2008). The primary alcohol reason for hospital attendance is alcohol withdrawal syndrome (AWS) which is a risk in people who have alcohol dependence syndrome (ADS) when they stop drinking (Vardy et al., 2017). ADS is recognised by the International Classification of Disease- Tenth Revision (ICD-10) when a person experiences three from a suite of six symptoms in the previous 12 months (World Health Organisation, 2016).

AWS and ADS span a spectrum that range from mild to severe, where mild withdrawal requires minimum intervention, and severe alcohol withdrawal syndrome (SAWS) that includes alcohol related seizures (ARS) and delirium tremens (DT's) hospital admission (Sutton \& Jutel, 2016; Feeney et al., 2015; Passeti et al., 2008). Although SAWS is experienced by approximately $10 \%$ of people, emergency department physicians are under pressure to see patients and make decisions quickly (Vardy et al., 2017). Consequently, a high proportion of patients are admitted to hospital acutely for alcohol detoxification incase they develop SAWS. The result of this just incase practice is that patients are discharged after 24 hours and has contributed to the increase in short to zero stay hospital admissions (ISD 
Scotland, 2017; NHS England, 2016; Maldonado et al., 2015; Pecoraro et al., 2013: Dolman \& Hawkes, 2005).

The decision to admit to hospital for alcohol detoxification is not supported by the lack of a tool to inform physicians in this decision (Benson et al., 2019; NICE, 2016; Stephens et al., 2014; Repper-DeLisi et al., 2008). Despite a number of retrospective studies investigating risk factors and SAWS, the only protocol located for the identification of low SAWS risk was developed by Stephens et al. (2014). The Clinical Institute Withdrawal Assessment- Alcohol Revised (CIWA-AR) (Sullivan et al., 1989) was central to the Stephens et al. (2014) protocol where SAWS is suspected at a CIWA-AR $>15$. While CIWA-AR $>15$ necessitated inpatient detoxification, patients who had a CIWA-AR $<15$ were screened for discharge (Stephens et al., 2014).

Detoxification carried out in hospital is up to eight times more costly than outpatient treatment, and reduces the likelihood that patients will attend alcohol treatment programmes on hospital discharge (Alwyn et al., 2004; Cooper, 1995; Bartu \& Saunders, 1994). Importantly, outpatient detoxification presents comparable results with inpatient detoxification for patient abstinence, engagement, satisfaction and adverse events (Passeti et al., 2008). However, the high incidence of alcohol presentations and subsequent admissions to hospital are having a detrimental impact on the NHS ability to achieve the Government's waiting time target (ISD Scotland, 2017; NHS England, 2016). In NHS Greater Glasgow \& Clyde (NHSGGC), the guarantee that $95 \%$ of patients will be admitted or discharged within four hours of their presentation to the emergency department is achieved in less than $90 \%$ of cases (ISD Scotland, 2017). 
NHSGGC provides health care to a population of 1.2 million people and covers six of the most deprived post code areas in Scotland. Deprivation in Scotland is captured by the Statistical Index of Multiple Deprivation where 1 is the most deprived and 5 the least (ISD Scotland, 2017). People living in the most deprived post code areas are eight times more likely to attend the emergency department for an alcohol related condition than people from more affluent areas (ISD Scotland, 2017; Walsh et al., 2010; The Scottish Government, 2009). In 2015/16 there were approximately 12,000 alcohol related acute hospital admissions in Glasgow (ISD Scotland, 2017). Patients in NHSGGC admitted with an alcohol problem are referred to the acute addiction liaison nursing service (AALNS). The AALNS are a team of specialist nurses who provide assessment, treatment planning and onward referral for problematic alcohol use patients admitted to hospital (McPherson \& Benson, 2011). In NHSGGC, the level of a person's alcohol consumption and AWS symptoms are captured by the Glasgow Assessment Management of Alcohol (GAMA) guideline (McPherson et al., 2012).

The GAMA is a comprehensive guideline that includes screening for alcohol use and dependency using the Fast Alcohol Screening Test (FAST) and a simple numeric score, the Glasgow Modified Alcohol Withdrawal Scale (GMAWS) to assess the symptoms of AWS (McPherson et al., 2012). The original GAMA study (McPherson et al., 2012) like studies by Maldonado et al. (2015), Pecoraro et al. (2013) and Dolman \& Hawkes (2005) developed the GMAWS to identify escalating withdrawal symptoms for the purpose of hospital treatment and not early discharge. The GAMA suggests ADS at FAST greater or equal ( $\geq$ ) 9 and severe ADS at FAST $\geq 12$ (McPherson et al., 2012). In addition, mild- moderate AWS is reflective of GMAWS $<4$ and SAWS, GMAWS $\geq 4$ (McPherson et al., 2012). The GMAWS and 
FAST have been validated and tested previously in the NHSGGC hospitals (McPherson et al., 2012).

The aim of this study and described in this paper is to investigate whether the GMAWS and the quantitative variables identified through a systematic literature review (Benson et al., 2019) can be used in the emergency department to help stratifying a person's risk of developing SAWS and support clinicians in their decision to admit or not. The variables were; previous SAWS, ARS, DT`s, hours since last drink, age, post code, gender, reason for admission, physical observations, blood results, co-morbidities, and level of ADS. Currently only one study has explored the use of an alcohol risk stratification tool for the purpose of emergency department discharge (Stephens et al., 2014). Because SAWS development is a consequence of years of drinking we employed a retrospective method, where risk measurement is time from data collection to analysis.

\section{METHODS}

\section{Patients}

Patients admitted acutely to the two large Glasgow acute adult hospitals within NHSGGC; Queen Elizabeth University Hospital (QEUH) and Glasgow Royal Infirmary (GRI) through the emergency department and referred to the AALNS during one calendar month ( $1^{\text {st }}$ April $30^{\text {th }}$ April 2016) were retrospectively included in the study. This month was chosen because the study was part of a time limited project that coincided with the end of this period. All patients were treated using the GAMA guideline and benzodiazepines administered for AWS symptoms, and thiamine for treatment and prophylaxis of alcohol related brain damage. ADS 
was suspected at FAST $\geq 9$ and confirmed using ICD-10 classification, while SAWS was recognised as GMAWS $\geq 4$. Patients were only counted once and subsequent admissions excluded. We also excluded patients who had no documented ICD-10 classification and past or present alcohol history. Ethical approval was granted by the NHS West of Scotland Research Ethics Committee (16/WS/0194).

\section{Data collection}

The medical records of the potential cohort for the study period were reviewed for exclusion criteria by a single investigator (AM) using a standard extraction form. The inclusion process is shown in Figure 1. Four hundred and eighty nine records related to 432 patients, with only the first referral for patients retained. The first admission was used as this patient group are frequently admitted to hospital and in a number of occasions this may be recent, and therefore prior treatment could adversely impact on the patients presenting GMAWS. Of the 432 records only 400 had complete data, which also included ICD-10 classification of ADS. Next, a second investigator (GB) reviewed the records and recorded the variables of interest identified by the systematic literature review. The GMAWS measured the patients SAWS status (GMAWS 0-10) on attendance at the emergency department and during admission. SAWS was reflective of GMAWS $\geq 4$ and no SAWS, GMAWS $<4$ and was supported by the total benzodiazepine requirement throughout admission. Any discrepancies were resolved by the wider research team. 


\section{Data Analysis}

Data was analysed using the Statistical Package for Social Scientists (SPSS) programme version 22. Spearman`s rank correlation coefficient was used to understand the unique contribution of the independent variables such as systolic blood pressure (SBP) on the dependent variable SAWS status (highest GMAWS). Additionally, as Spearman`s rank correlation coefficient does not make assumptions about the distribution of the data it was a test recommended when using an ordinal dependent variable such as GMAWS. Standard multiple linear regression and stepwise regression was employed to determine how the addition of each statistically significant variable $(p=<0.05)$ contributed to the patients SAWS status during admission, while controlling for all others. Finally, as the aim of the study was to develop a tool to identify low risk of SAWS, there was a need to differentiate between SAWS (GMAWS $\geq 4$ ) and no SAWS (GMAWS $<4$ ). Therefore, binary logistic regression was performed on the variables to determine how effective they were in predicting the final SAWS status based on a dichotomised outcome of SAWS versus no SAWS. Binary logistic regression identified the sensitivity, specificity, positive predictive value (PPV) and negative predictive value (NPV) of the variables that made the greatest contribution to the patients SAWS status during admission.

\section{RESULTS \\ Cohort $(n=400)$}

We collated the records from 400 patients, where 210 (53\%) did not develop SAWS during their admission. Table 1 shows that the majority of patients were White Scottish $(355 / 400)$ and the mean age of the cohort was $50( \pm 12)$ years. The cohort was predominantly male (283 males, 117 females) and co-morbidity was recorded in 212 patient records and more 
prevalently recorded for patients who did not develop SAWS. Alcohol liver disease (ALD) was the most prominent $(n=78)$ co-morbidity in the cohort and almost three quarters of patients lived in the two most deprived areas $(n=337)$. The alcohol related conditions of AWS $(n=190)$ and ARS $(n=45)$ were the most common reason for admission and self-harm $(n=29)$ the most non-alcohol related reason.

The mean FAST for the cohort was $(14 \pm 1.8)$ while over one third of the cohort records had a previous history of ARS recorded $(\mathrm{n}=138)$. The mean time since the cohorts last alcoholic drink was $30( \pm 20)$ hours and mean alcohol units consumed approximately $196( \pm 81)$ per week. The percentage of males who developed SAWS was greater than those that did not develop SAWS (142/190, 75\% versus (vs) 141/210, 67\%) which was reversed for females (48/190, 25\% vs 69/210, 33\%). The group that developed SAWS tended to be slightly younger (49 vs 51 years).

Table 1 shows that of the blood results alanine aminotransferase (ALT) $(r=0.244, \mathrm{~N}=398$, $p<0.01)$, aspartate aminotransferase $(\mathrm{AST})(r=0.209, \mathrm{~N}=398, p<0.01)$ and albumin $(r=$ $0.239, \mathrm{~N}=398, p<0.01)$ were significant and positively correlated with SAWS status during admission although accounted for only $6 \%, 4 \%$, and $6 \%$ of the outcome. In addition, potassium $(r=-0.110, \mathrm{~N}=398, p<0.05)$ was negatively correlated and responsible for $1 \%$ of the SAWS status. The physiological markers of systolic blood pressure (SBP) $(r=0.427$, $\mathrm{n}=398, p<0.01)$, and pulse $(\mathrm{r}=0.136, \mathrm{n}=398, p<0.01)$ explained $18 \%$ and $2 \%$ of the SAWS status. 
FAST $(r=0.243, \mathrm{~N}=398, p<0.01)$, GMAWS $(r=0.822, \mathrm{~N}=398, p<0.01)$ score at the emergency department, and hours since last drink $(r=0.548, \mathrm{~N}=398, p<0.01)$ were positively correlated with the SAWS status experienced during admission, while previous SAWS $(r=-$ 0.117, $\mathrm{N}=398, p<0.05)$ and ARS $(r=-0.198, \mathrm{~N}=398, p<0.05)$ negatively correlated. Spearman`s rank correlation identified that in isolation these variables were responsible for $1 \%$ (SAWS) to $68 \%$ (GMAWS) of the variance in a patients SAWS status during hospital admission.

The variables, hours since last drink, FAST, GMAWS at the emergency department, previous ARS, SAWS, ALT, AST, potassium, albumin, SBP, and pulse where analysed further using standard multiple linear regression. The standard multiple linear regression $\mathrm{R}$ value for the 11 variables above was strong $(0.843)$ and accounted for an adjusted $\mathrm{R}^{2}$ (squared) that explained $71 \%$ of the SAWS status experienced by patients during their hospital admission: F $(11,396)$ $=72.83, p<0.001$. However, using stepwise regression, seven of the variables were removed as they were not identified as predictor variables: ARS, SAWS, ALT, AST, potassium, albumin, and pulse. A significant model emerged that included: hours since last drink, FAST, GMAWS, and SBP, F $(4,396)=234.12, p<0.001$. Table 2 shows that although the total $\mathrm{R}^{2}$ adds up to $70 \%$, its components are not unique, where fitting the selected predictors in a different order will result in different individual contribution to the same total $\mathrm{R}^{2}$ outcome.

A logistic regression analysis was performed with SAWS status $(\mathrm{SAWS}=0$, no SAWS $=1$ ) as the dependent variable and GMAWS at the emergency department, hours since last drink, FAST and SBP as predictor variables. A total of 400 cases were analysed and the total model significantly predicted SAWS status (omnibus chi-square $=316.68$, df- $4, p<0.0005$ ). The 
model accounted for between $54.7 \%$ and $73.0 \%$ of the variance in SAWS status, with $89 \%$ of patients who developed SAWS and $84 \%$ of patients not developing SAWS correctly predicted. Overall, $87 \%$ of predictions were accurate. Table 3 shows that each increase in the GMAWS by a score of one is associated with a decrease in the odds of no SAWS by a factor of $2.76(95 \%$ CI $2.21,3.45)$. In addition, increasing the time since last drink by 1 hour, FAST by a score of 1 and SBP by $1 \mathrm{mmHg}$ increase the odds of SAWS by 1.31 (95\% CI $1.24,1.37), 1.30$ (CI 1.08, 1.57) and 1.22 (95\% CI 1.10, 1.34) respectively.

The study identified that the risk of a patient developing SAWS could be recognised at the emergency department with GMAWS $\geq 4$, FAST $\geq 15$, SBP $>138 \mathrm{mmHg}$ and hours since last drink $<44$ hours (Table 1) and therefore the risk of not developing SAWS with GMAWS $<4$, FAST $<15, \mathrm{SBP} \leq 138 \mathrm{mmHg}$ and hours since last drink $\geq 44$ hours.

\section{DISCUSSION}

This retrospective cohort study was conducted to capture a population of patients who had a prevalent diagnosis of ADS and admitted to two large acute adult hospitals in Glasgow. A consequence of ADS is the development of AWS on cessation of alcohol use. AWS spans a spectrum that ranges from mild requiring minimal intervention to severe, where hospital admission and pharmacological treatment is required (Sutton \& Juttel, 2016). Although there are tools for the treatment of AWS, their purpose is for the identification and treatment of those at high risk of SAWS (Maldonado et al., 2015; Pecoraro et al., 2013; McPherson et al., 2012: Dolman \& Hawkes, 2005). The studies investigating AWS identified that the emergency department was a good location to assess SAWS risk and commence treatment 
(Maldonado et al., 2015; Pecoraro et al., 2013; Eyer et al., 2011; Monte et al., 2010; Mennecier et al., 2008; Wright et al., 2006; Lee et al., 2005; Dolman \& Hawkes, 2005; Lukan et al., 2002; Ferguson et al., 1996; Booth \& Blow, 1993). While we agree that the emergency department is an ideal location to identify risk, we looked at assessment as an opportunity to identify and discharge patients who had ADS and at low risk of SAWS.

\begin{abstract}
Attendance at the emergency department with AWS is an ideal opportunity to develop a noninvasive measurement to stratify SAWS risk and discharge low risk patients home. From this study, a combination of GMAWS at the emergency department, FAST, hours since last drink and SBP appear to be a potential indicator of SAWS risk with sensitivity classification showing $84 \%$ accuracy in ruling out those without the disease, and $89 \%$ specificity ruling in those with the disease.
\end{abstract}

The results of our study are comparable with the studies by Maldonado et al. (2015), Pecoraro et al. (2013) and Dolman and Hawkes (2005), although these studies looked at risk stratification for hospital treatment. While the Maldonado et al. (2015) tool comprised of the 10 most common factors identified from their systematic literature review (Maldonado et al., 2014), Goodson et al. (2014) in their meta-analysis of 15 studies found that only ARS and DT's where statistically significant in SAWS risk in more than one study. The lack of consistent variables from studies investigating the same phenomenon suggests that the link with SAWS was either a chance association or a consequence of poor study design, execution or low prevalence. Moreover, although Pecoraro et al. (2013) and Dolman and Hawkes (2005) propose that the Alcohol Use Disorder Identification Test (AUDIT) was effective in identifying a person's risk of SAWS, this claim was not supported in other studies (Von-der- 
Pahlen et al., 2008; Shevlin \& Smith, 2007; Lima et al., 2005). In contrast to our study these published risk stratification tools were based on positive results from small prevalence samples (7\%; 1.4\%; 2\%) (Maldonado et al., 2015; Pecoraro et al., 2013; Dolman \& Hawkes 2005).

The one study by Stephens et al. (2014) that looked at low SAWS risk, unlike our study presented a limited systematic literature review that included only three studies. The key to the Stephens et al. (2014) process was the CIWA-AR and like the GMAWS presented scores for mild- moderate AWS and SAWS, where the mild to moderate patients were discharged home. Previous research has identified that discharge and successful engagement with alcohol care and treatment services through a home supported detoxification has been associated with a $59 \%$ reduction in emergency department attendances and $66 \%$ reduction in frequent hospital admissions (Tadros et al., 2013; Hughes et al., 2013). However, the Stephens et al. (2014) process provided an outpatient detoxification that was unsupported. In addition, this process was guided by the CIWA-AR were the predictability for stratifying low SAWS risk was not investigated, and therefore risk of adverse events on discharge, unknown. Consequently, the number of readmissions within the study population increased. For this reason and recognition that the variables in our tool were unable to guarantee no risk, an alternative to hospital admission in the form of a home supported detoxification for the population discharged from hospital would be explored.

In the AWS literature the importance of ADS appears to be overlooked, and presented as a homogenous concept captured as a single measure by the International Classification of Disease (ICD) and the Diagnostic and Statistical Manual of Mental Disorders (DSM) 
(Maldonado et al., 2015; Pecoraro et al., 2013; Eyer et al., 2011; Monte et al., 2010; Mennecier et al., 2008; Wright et al., 2006; Lee et al., 2005; Dolman \& Hawkes, 2005; Lukan et al., 2002; Ferguson et al., 1996; Booth \& Blow, 1993). However, like AWS, ADS spans a spectrum where SAWS risk is proportionate to a person's level of alcohol dependence (Stewart \& Swain, 2012). Therefore, as a determinant of SAWS the measure of ADS severity is paramount as a starting point to understanding risk (Stockwell et al., 1983). Subsequently, none of the studies reviewed used a tool to record the severity of the persons ADS in an environment where poor alcohol assessment is known (Sutton \& Juttel, 2016; Stehman \& Mycyk, 2013; Forsythe \& Lee, 2012). Consequently, it is difficult to exclude the prospect that the results and low prevalence of ADS may be as reflective of the quality of assessment data as their association with SAWS risk.

Our study, unlike the AWS literature (Maldonado et al., 2015; Pecoraro et al., 2013; Eyer et al., 2011; Monte et al., 2010; Mennecier et al., 2008; Wright et al., 2006; Lee et al., 2005; Dolman \& Hawkes, 2005; Lukan et al., 2002; Ferguson et al., 1996; Booth \& Blow, 1993), provided a population where the prevalence of ADS was high. The prevalence of ADS and use of FAST as a measure of ADS severity provided a population deemed high risk of SAWS. Consequently, the novel concept attributed to this study and not found in the literature is the development of a research based tool that can stratify a person's risk of developing SAWS and support clinicians in their decision to admit or discharge low risk patients from the emergency department.

The strengths of this study are the accuracy of the data collected by a specialist team of alcohol nurses. The retrospective nature of the study supported that GMAWS, FAST, hours 
since last drink and SBP were not influenced by the aim of the study. The use of a high alcohol screening score (FAST $\geq 15$ ) and high withdrawal symptom score (GMAWS $\geq 4$ ) seem like reasonable indicators of SAWS risk (McPherson et al., 2012) and therefore FAST < 15 and GMAWS $<4$ indicators of low SAWS risk. While time since last drink as an indicator of SAWS risk divides the literature (Maldonado et al. 2015; Eyer et al., 2011; Lee et al., 2005; Lukan et al., 2002; Ferguson et al., 1996) it is safe to assume that SAWS risk decreases with the increase of time (Munchie et al., 2013). However, the impact of SBP is less clear in this population where high levels of co-morbidity and subsequent pharmacological treatment may have normalised an otherwise abnormal SBP (Chen et al., 2015).

Despite the strengths of this study there are a number of limitations. First, the study was conducted in a single healthcare system that has a high prevalence of ADS, although the indicators within the tool are generalisable to other populations. Second, the original GAMA study (McPherson et al., 2012) presented high risk of SAWS at FAST $\geq 12$, which may have resulted in some low risk patients receiving pharmacological intervention influencing their SAWS status. Finally, while the risk stratification tool had excellent predictability, the included variables were not fully responsible for the variance in whether the patient developed SAWS or not, and therefore an alternative treatment pathway is suggested in the form of a home supported detoxification.

The developed risk stratification tool shows considerable promise as a predictor of low risk of SAWS and safe discharge from the emergency department. In addition, the risk stratification tool includes variables that are collected routinely as part of a patient's emergency department journey. However, further research into the use of this risk stratification tool and 
the predictor variables are required and should include a prospective cohort study utilising a home supported detoxification as an alternative to hospital admission for low risk patients. 


\section{REFERENCES}

Alwyn, T., John, B., Hodgson, R. J. And Phillips, C. J. (2004). The Addition of Psychological Intervention to a Home Detoxification Programme. Alcohol \& Alcoholism, 39 (6): 536-541.

Bartu, A. And Saunders, W. (1994). Domiciliary Detoxification: A Cost Effective Alternative to Inpatient Treatment. The Australian Journal of Advanced Nursing, 11 (4): 12-18.

Benson, G., Roberts, N., McCallum. J. And McPherson, A. (2019). Severe Alcohol

Withdrawal Syndrome: Review of the Literature. Drugs and Alcohol Today. Available from https://doi.org/10.1108?DAT-10-2018-0051.

Booth, B.M, \& Blow, .FC., (1993). The Kindling Hypothesis: Further Evidence from a U.S. National Survey of Alcoholic Men. Alcohol and Alcoholism.28 (4): 593-598.

Chen, P., Chaugai, A., Zhao, F. and Wang, D. W. (2015). Cardioprotective Effect of Thiazide-Like Diuretics: A Meta-Analysis. American Journal of Hypertension, 28 (12): $1453-1463$.

Cooper, D. B. (1995). Alcohol Home Detoxification: A Way Forward. British Journal of Nursing. 4 (22): 1315-1318.

The Department of Health., (2008). The Cost of Alcohol Harm to the NHS in England. London: Department of Health.

Dolman, J. M. \& Hawkes, N. D., (2005). Combining the Audit Questionnaire and Biochemical Markers to Assess Alcohol Use and Risk of Alcohol Withdrawal in Medical Inpatients. Alcohol Alcohol, 40 (6): 515-519. 
Eyer, F., Schuster, T., Felgenhauer, N., Pfab, R., Strubel, T., Saugel, B, \& Zilker, T., (2011). Risk Assessment of Moderate to Severe Alcohol Withdrawal. Alcohol Alcoholism. 46 (4): $427-433$.

Feeney, C., Harrison, A., Eike, J., Mathew, R., Shirley, S., Indhu, S. \& Carter, C. R., (2015). A Simplified Protocol for the Treatment of Alcohol Withdrawal. Journal of Addiction Medicine, 9 (6): 485-490.

Ferguson, J.A., Suelzer, C.J., Eckert, G.J., Zhou, X.D., Dittus, R.S, \& So, J., (1996). Risk Factors for Delirium Tremens Development. Journal of General Internal Medicine. 11: 410414.

Forsythe, M. \& Lee, G. A., (2012). The Evidence for Implementing Alcohol Screening and Intervention in the Emergency Department- Time to Act. International Emergency Nursing, 20 (3): 167-172.

Goodson, C. M., Clark, B. J. \& Douglas, I. S., (2014). Predictors of Severe Alcohol Withdrawal Syndrome: A Systematic Review and Meta- Analysis. Alcoholism Clinical and Experimental Research, 38 (10), 2664-2677.

Holman, C. D., English, D. R., Milne, E. \& Winter, M. G., (1996). Meta-analysis of Alcohol and All-Cause Mortality: A Validation of NHMRC Recommendations. Medical Journal of Australia, 164 (3), 141-145.

Hughes, N. R., Houghton, N., Nadeem, H., Bell, J., McDonald, S., Glynn, N., Scarfe, C., MaCkay, B., Rogers, A., Walters, M. Smith, M., McDonald, A. \& Dalton, D., (2013). Salford Assertive Outreach Team: A New Tool for Reducing Alcohol Related Admissions. Frontline Gastroenterology, 4 (2), 130-134. 
Information Services Division (ISD) Scotland., (2017). Information Service Division. Available from www.isdscotland.org.

Lee, J.H., Jang, M.K., Lee, J.Y., Kim, S.M., Kim, K.H., Park, J.Y, Lee, J.H., Kim. H.Y, \& Yoo, J.Y., (2005). Clinical Predictors for Delirium Tremens in Alcohol Dependence. Journal of Gasrtoenterology Hepatology. 20 (12): 1833-1837.

Lima, C. T., Freire, A. C., Silva, A. P., Teixeira, R. M., Farrel, M. \& Farrel, M., (2005). Concurrent and Construct Validity of the Audit in Urban Brazilian Sample. Alcohol and Alcoholism, 40 (6), 584-589.

Lukan, J.K., Reed, D.N., Looney, SW., Spain, D.A, \& Blondell, R.D., (2002). Risk Factors for Delirium Tremens in Trauma Patients. Journal of Trauma. 53 (5): 901-906.

Maldonado, J. R., Sher, Y. \& Ashouri, J. F., (2014). The "Prediction of Alcohol Withdrawal Severity Scale" (PAWSS): Systematic Literature Review and Pilot Study of a New Scale for the Prediction of Complicated Alcohol Withdrawal Syndrome. Alcohol, 48 (4), 375-90.

Maldonado, J.R., Sher, Y., Das, S., Hills-Evans, A.F., Lolak, S., Talley, R, \& Neri, E., (2015). Prospective Validation of the Prediction of Alcohol Withdrawal Severity Scale (PAWSS) in Medically Ill Patients: A New Scale for the Prediction of Complicated Alcohol Withdrawal Syndrome. Alcohol and Alcoholism. 50 (5): 1-10.

Manasco, A., Chang, S., Larriviere, J., Hamm, L. L. \& Glass, M., (2012). Alcohol Withdrawal. Southern Medical Journal, 105 (11): 607-612.

McPherson, A. \& Benson, G. (2011). The Development and Explication of a Unique NurseLed Addiction Liaison Service for the Acute Hospitals in the Greater Glasgow Area. Drugs and Alcohol Today, 11 (1): 18-25. 
McPherson, A., Benson, G. \& Forrest, E.H., (2012). Appraisal of the Glasgow and Management of Alcohol Guideline: A Comprehensive Alcohol Management Protocol for use in General Hospitals. Quarterly Journal of Medicine, 105 (7): 649-56.

Mennecier, D., Thomas, M., Arvers, P., Corberand, D., Sinayoko, L., Bonnefoy, S., Harnois, F., \& Thiolet, C. (2008) Factors Predictive of Complicated or Severe Alcohol Withdrawal. Gastroenterology Clinical Biology 32 (8): 792-797.

Monte, R., Rabunai, R, \& Casariego, E., (2010). Analysis of the Factors Determining Survival of Alcoholic Withdrawal Syndrome Patients in a General Hospital. Alcohol and Alcoholism. 45 (2):151-158.

Munchie, H.L., Yasinian, Y, \& Oge, L., (2013). Outpatient Management of Alcohol Withdrawal Syndrome. American Family Physician. 88 (9): 589-595.

NHS England., (2016). A\&E Attendances and Emergency Admissions. Available from https://www.england.nhs.uk/statistics/statistical-work-areas/ae-waiting-times-and-activity/

National Institute for Health \& Clinical Excellence (NICE). (2016). Acute Alcohol

Withdrawal. Available from http://www.pathways.nice.org.uk/pathways/alcohol-usedisorders.

Passetti, F., Jones, G., Chawla, K., Bowland, B. And Drummond, C. (2008). Pilot Study of Assertive Community Ttreatment Methods to Engage Alcohol-Dependent Individuals. Alcohol Alcoholism, 43 (4), 451-455.

Pecoraro, A., Ewen, E., Horton, T., Mooney, R., Kolm, P., McGraw, P. \& Woody G., (2013). Using the AUDIT-PC to Predict Withdrawal in Hospitalized Patients. Journal of General International Medicine, 29 (1): 7-9. 
Repper-Delisi, J., Stern, T. A., Mitchell, M., Lussier-Cushing, M., Lakatos, B., Fricchione, G, L., Quinlin, J., Kane, M., Berube, R., Blais, M., Capasso, V., Pathan, F., Karson, A. and Bierber, M. (2008). Successful Implementation of an Alcohol-Withdrawal Pathway in a General Hospital. Psychosomatics, 49 (4): 292-299.

The Scottish Government., (2009). Changing Scotland's Relationship with Alcohol: A Framework for Action. Edinburgh: The Scottish Government.

Shevlin, M. \& Smith, G. W., (2007). The Factor Structure and Concurrent Validity of the Alcohol Use Disorders Identification Test based on a Nationally Representative UK Sample. Alcohol and Alcoholism, 42, 582-587.

Stehman, C. R. \& Mycyk, M. B., (2013). A Rational Approach to the Treatment of Alcohol Withdrawal in the ED. American Journal of Medicine, 31 (4): 734-742.

Stephens, J.R., Liles, A., Dancel, R., Gilchrist, M., Kirsch, J, \& De Walt, D.A., (2014). Who Needs Inpatient Detox? Development and Implementation of a Hospitalist Protocol for the Evaluation of Patients for Alcohol Detoxification. Journal of Internal General Medicine. 29 (4): 587-593.

Stewart, S. and Swain, S. (2012). Assessment and Management of Alcohol Dependence and Withdrawal in the Acute Hospital. Clinical Medicine, 12 (3): 266-271.

Stockwell, T., Murphy, D. \& Hodgson, R., (1983). The Severity of Alcohol Dependence Questionnaire: Its Use, Reliability and Validity. British Journal of Addiction, 78 (2), 145155.

Sullivan, J, T., Sykora, K., Schneiderman, J., Naranjo, C. A. and Sellers, E.M. (1989). Assessment of Alcohol Withdrawal: The Revised Clinical Institute Withdrawal Assessment of Alcohol withdrawal: The revised Clinical Institute Withdrawal Assessment for Alcohol 
Scale (Clinical Institute Withdrawal Assessment-Alcohol revised). British Journal of Addiction, 84 (11): 1353-1357.

Sutton, L.J, \& Jutel, A., (2016). Alcohol Withdrawal Syndrome in Critically Ill Patients: Identification, Assessment, and Management. Critical Care Nurse. 36 (1): 28-39.

Tadros, G., Salama, R. A., Kingston, P., Mustafa, N., Johnson, E., Pannell, R., \& Hashmi, M., (2013). Impact of an Integrated Rapid Response Psychiatric Liaison Team on Quality Improvement and Cost Savings: The Birmingham RAID Tool. Psychiatrist, 37 (1), 4-10.

Vardy, J., Keliher, T., Fisher, J., Ritchie, F., Bell, C., Chekroud, M., Clarey, F., Blackwood, L., Barry, L., Paton, E., Clark, A. \& Connelly, R., (2017). Quantifying Alcohol-Related Emergency Admissions in a UK Tertiary Referral Hospital: A Cross-Sectional Study of Chronic Alcohol Dependency and Acute Alcohol Intoxication. British Medical Journal Open. Available from http://bmjopen.bmj.com.

Von-der-pahlen, B., Santtila, P., Witting, K., Witting, K., Vatjonen, M., Jern, P., Johansson, A. \& Sandnabba, N. K., (2008). Factor Structure of the Alcohol Use Disorders Identification Test (AUDIT) for Men and Women in Different Age Groups. Journal of Studies on Alcohol and Drugs, 69 (4), 616-621.

Walsh, D., Bendel, N., Jones, R. \& Hanlon, P., (2010). Its Not “Just Deprivation”: Why Do Equally Deprived UK Cities Experience Different Health Outcomes? Public Health. (online). Available from: www.phru.net/.../Glasgow\%20Effect\%20Paper\%20pub\%20health\%20March\%202010.pdf. Waye, C., Wong, M. \& Lee, S., (2015). Implementation of a CIWA-Ar Alcohol Withdrawal Protocol in a Veterans Hospital. Southern Medical Journal, 108 (1): 23-28. 
World Health Organisation., (2014). Global Status Report on Alcohol. Geneva: World Health Organisation.

World Health Organisation., (2016). International Classifications of Diseases- $11^{\text {th }}$ Revision. Available from https://www.icd.who.int/browse10/2016/en/.

Wright, T., Myrick, H., Henderson, S., Peters, H. and Malcolm, R. (2006). Risk Factors for Delirium Tremens: A Retrospective Chart Review. The American Journal on Addictions, 15 (3), 213-219. 
The manuscript is submitted as research. The corresponding author is George Benson, Dykebar Hospital, Grahamston Rd, Paisley, PA2 7DE. Emailgeorge.benson2@ggc.scot.nhs.uk.Telephone 07984178058.

'This paper contains original material and has not been, nor will be, published in whole or in part by any other journal'. Each author has contributed significantly to the work and gives consent for publication of the article Development of an Alcohol Withdrawal Risk Stratification Tool based on Patients Referred to an Addiction Liaison Nursing Service in Glasgow

No author has a conflict of interest with regards the paper and no external funding was acquired in producing this paper.

This research adds to the knowledge surrounding severe alcohol withdrawal syndrome (SAWS) risk. The research proposes that there are variables that can be captured routinely in the emergency department and developed a risk stratification tool based on these variables to support the safe discharge of low risk patients.

The authors

George Benson G Bersa.

Andrew McPherson lades ha hor

Jacqueline McCallum

$$
\text { Vacquetio Micalle }
$$

Nicola J Roberts A Robos 
AALNS

ADS

ALD

ALT

ARS

AST

AUDIT

AWS

CI

CIWA-Ar

DSM

DTs

FAST

GRI

GMAWS

GAMA

ICD-10

NHSGGC

NPV

OR

PAC

PPV

QEUH

SAWS

SBP

SPSS

\section{ABBREVIATIONS}

Acute Addiction Liaison Nursing Service

Alcohol Dependence Syndrome

Alcohol Liver Disease

Alanine aminotransferase

Alcohol Related Seizures

Aspartate aminotransferase

Alcohol Use Disorder Identification Test

Alcohol Withdrawal Syndrome

Confidence Interval

Clinical Institute Withdrawal Assessment- Alcohol Revised

Diagnostic and Statistical Manual of Mental Disorders

Delirium Tremens

Fast Alcohol Screening Test

Glasgow Royal Infirmary

Glasgow Modified Alcohol Withdrawal Scale

Glasgow Assessment Management of Alcohol

International Classification of Disease- Tenth Revision

National Health Service Greater Glasgow \& Clyde

Negative Predictive Value

Odds Ratio

Percentage Accuracy IN Classification

Positive Predictive Value

Queen Elizabeth University Hospital

Severe Alcohol Withdrawal Syndrome

Systolic Blood Pressure

Statistical Package for Social Scientists 
Figure 1 Algorithm for enrolment of patients for the cohort sample

Total Patients Referred to the Acute Addiction

Liaison Nursing Service who had a Fast Alcohol

Screening Test $($ FAST $) \geq 9(n=489)$

\section{Number of Excluded $(n=89)$ \\ Repeat admissions ( $n=57)$}

No International Classification of Disease diagnosis $(n=18)$

No hours since last drink $(n=5)$

No documentation of previous alcohol related history (Alcohol Related Seizure, Severe Alcohol Withdrawal Syndrome, Delirium Tremens) $(n=9)$

Total Patients Records included $(n=400)$ 
Table 1 Comparison of patients with and without development of SAWS

\begin{tabular}{|c|c|c|c|}
\hline & $\begin{array}{c}\text { SAWS } \\
(\mathrm{n}=190)\end{array}$ & $\begin{array}{c}\text { NO SAWS } \\
(n=210)\end{array}$ & $P$ value \\
\hline Male/ female & $142 / 48$ & $141 / 69$ & NS \\
\hline Mean age at admission (SD) & $48.6(10.54)$ & $50.8(12.8)$ & NS \\
\hline White Scottish N (\%) & $166(88)$ & $189(90)$ & NS \\
\hline White other British N (\%) & $15(8)$ & $17(8)$ & NS \\
\hline White Irish $\mathrm{N}(\%)$ & $1(0.5)$ & 0 & NS \\
\hline White Polish N (\%) & $6(3)$ & $2(1)$ & NS \\
\hline Asian N (\%) & $2(1)$ & $2(1)$ & NS \\
\hline $1^{\text {st }}$ quintile (most deprived) N (\%) & $121(64)$ & $137(65)$ & NS \\
\hline $2^{\text {nd }}$ quintile $N(\%)$ & $42(22)$ & $37(18)$ & NS \\
\hline $3^{\text {rd }}$ quintile $N(\%)$ & $14(7)$ & $17(8)$ & NS \\
\hline $4^{\text {th }}$ quintile $N(\%)$ & $9(5)$ & $9(4)$ & NS \\
\hline $5^{\text {th }}$ quintile (least deprived) N (\%) & $4(2)$ & $10(5)$ & NS \\
\hline Employed N (\%) & $14(7)$ & $15(7)$ & NS \\
\hline Alcohol Liver Disease (ALD) N (\%) & $33(16)$ & $45(24)$ & NS \\
\hline Cardiac N (\%) & $23(11)$ & $17((9)$ & NS \\
\hline Diabetes N (\%) & $14(7)$ & $24(13)$ & NS \\
\hline Cerebral Vascular Disease (CVD) N (\%) & $6((3)$ & $5(3)$ & NS \\
\hline Cancer N (\%) & $1(0.5)$ & $2(1)$ & NS \\
\hline Pancreatitis N (\%) & $18(9)$ & $13(7)$ & NS \\
\hline Head injury N (\%) & $5(2)$ & $6(3)$ & NS \\
\hline Reason for admission $\mathrm{N}(\%)$ & & & NS \\
\hline Alanine aminotransferase (ALT) (U/L) N (SD) & $71.4(132.67)$ & $53.3(82.30)$ & 0.0005 \\
\hline Aspartate aminotransferase (AST) (U/L) N (SD) & $108.9(163.41)$ & $82.9(106.02)$ & 0.0005 \\
\hline Potassium (mmol/l) N (SD) & $3.99(0.52)$ & $4.1(0.59)$ & 0.028 \\
\hline Platelets (X 10^9/L) N (SD) & $206.6(110.68)$ & $216.2(106.44)$ & NS \\
\hline Albumin $(\mathrm{g} / \mathrm{l}) \mathrm{N}(\mathrm{SD})$ & $36.8(5.89)$ & $34.6(6.40)$ & 0.0005 \\
\hline Fast Alcohol Screening Test (FAST) N (SD) & $15(1.6)$ & $14(1.8)$ & 0.0005 \\
\hline $\begin{array}{l}\text { Previous history of Severe alcohol withdrawal } \\
\text { syndrome (SAWS) ever N (\%) }\end{array}$ & $50(26)$ & $41(20)$ & 0.019 \\
\hline Previous history of alcohol related seizure (ARS) & & & \\
\hline 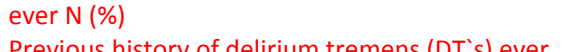 & $80(42)$ & $58(28)$ & 0.0005 \\
\hline $\begin{array}{l}\text { Previous history of delirium tremens (DT s) ever } \\
\mathrm{N}(\%)\end{array}$ & $18(9)$ & $15(7)$ & NS \\
\hline Hours since last drink N (SD) & $44.1(21.66)$ & $17.6(15.75)$ & 0.0005 \\
\hline Systolic Blood Pressure (SBP) (mmHg) N (SD) & $138.4(18.39)$ & $125.1(19.86)$ & 0.0005 \\
\hline Pulse (bpm) N (SD) & $99.4(10.54)$ & $94.4(20.04)$ & 0.006 \\
\hline
\end{tabular}

Data are expressed as mean and standard deviation (SD) for continuous variables and as frequency ( $n$. \%) for categorical variables. Only Significant $P$ values $(p<0.05)$ are presented.

Severe alcohol withdrawal syndrome (SAWS) was determined as Glasgow Modified Alcohol Withdrawal Scale (GMAWS) $\geq 4$ and no SAWS, GMAWS $<4$ 

regression)

\begin{tabular}{|c|c|c|c|c|c|c|c|}
\hline \multirow[b]{2}{*}{ Model } & \multicolumn{2}{|c|}{$\begin{array}{c}\text { Unstandardised } \\
\text { Coefficients }\end{array}$} & \multirow{2}{*}{$\begin{array}{l}\begin{array}{c}\text { Standardised } \\
\text { Coefficients }\end{array} \\
\text { Beta }\end{array}$} & \multirow[t]{2}{*}{ sig } & \multicolumn{2}{|c|}{$\begin{array}{l}\text { 95\% Confidence } \\
\text { Intervals for B }\end{array}$} & \multirow[t]{2}{*}{ R square } \\
\hline & B & $\begin{array}{l}\text { Std. } \\
\text { Error }\end{array}$ & & & Lower & Upper & \\
\hline $\begin{array}{l}\text { Glasgow Modified Alcohol } \\
\text { Withdrawal Scale } \\
\text { (GMAWS) (1) }\end{array}$ & 0.665 & 0.035 & 0.660 & 0.0005 & 0.596 & 0.733 & $66 \%$ \\
\hline Hours since last drink (2) & 0.015 & 0.003 & 0.166 & 0.0005 & 0.009 & 0.021 & $+1(68) \%$ \\
\hline $\begin{array}{l}\text { Systolic blood pressure } \\
\text { (SBP) (3) }\end{array}$ & 0.13 & 0.004 & 0.131 & 0.001 & 0.006 & 0.021 & $+1+2(69 \%)$ \\
\hline $\begin{array}{l}\text { Fast Alcohol Screening } \\
\text { Test (FAST) (4) }\end{array}$ & 0.083 & 0.035 & 0.071 & 0.018 & 0.014 & 0.152 & $+1+2+3(70 \%)$ \\
\hline
\end{tabular}

Dependent variable was highest Glasgow Modified Alcohol Withdrawal Scale (GMAWS) score (0-10). The $R$ square represents variables identified using stepwise multiple regression. The order and percentage contribution to the models predictive ability is presented 
Table 3 Multivariate logistic regression model. Independent clinical predictors in patients SAWS status (highest GMAWS)

\begin{tabular}{|l|l|l|l|l|l|}
\hline & & & \multicolumn{3}{|l|}{$95 \%$ Cl for OR } \\
\hline Model & $B$ & sig & OR & Lower & Upper \\
\hline $\begin{array}{l}\text { Glasgow Modified } \\
\text { Alcohol Withdrawal } \\
\text { Scale (GMAWS) }\end{array}$ & 1.016 & 0.0005 & 2.762 & 2.210 & 3.450 \\
\hline Hours since last drink & 0.054 & 0.0005 & 1.31 & 1.24 & 1.37 \\
\hline $\begin{array}{l}\text { Systolic blood pressure } \\
\text { (SBP) }\end{array}$ & 0.022 & 0.014 & 1.22 & 1.10 & 1.34 \\
\hline $\begin{array}{l}\text { Fast Alcohol Screening } \\
\text { Test (FAST) }\end{array}$ & 0.265 & 0.006 & 1.30 & 1.08 & 1.57 \\
\hline
\end{tabular}

B, Beta, sig, significance, OR, Odds Ratio, Cl, Confidence Intervals 